\title{
Correction to: From Bayesian Inference to Logical Bayesian Inference
}

\author{
A New Mathematical Frame for Semantic \\ Communication and Machine Learning
}

Chenguang Lu 10

Correction to:

Chapter "From Bayesian Inference to Logical Bayesian Inference: A New Mathematical Frame for Semantic Communication and Machine Learning" in: Z. Shi et al. (Eds.): Intelligence Science II, IFIP AICT 539, https://doi.org/10.1007/978-3-030-01313-4_2

The original version of this chapter contained a mistake. There was an error in Equation (31). The original chapter has been corrected. 